\title{
Alternative Chemotherapy Schedules in Ewing's Sarcoma: An Indian Perspective
}

\author{
Pramod Kumar Singh ${ }^{1} \quad$ Rakesh Kapoor $^{1} \quad$ Ravi Teja $^{1} \quad$ Ritesh Kumar $^{1} \quad$ Amit Bahl $^{1}$ \\ ${ }^{1}$ Department of Radiation Oncology, Post Graduate Institute of \\ Medical Education and Research (PGIMER), Chandigarh, India

\begin{abstract}
Address of correspondence Pramod Kumar Singh, MBBS, MD, Department of Radiation Oncology, Post Graduate Institute of Medical Education and Research (PGIMER), Chandigarh 160012, India (e-mail: drpramodsingh16@gmail.com).
\end{abstract}

\begin{abstract}
Advances in the treatment of Ewing's sarcoma family of tumors (ESFTs) are a result of improvements in systemic and local therapies. VACA (vincristine, Adriamycin [doxorubicin], cyclophosphamide, dactinomycin) chemotherapy alone versus VACA + IE (VACA with alternate ifosfamide, etoposide) improve survival, but this regimen cannot be given to all patients due to poor tolerance and 5-day schedule chemotherapy in VACA + IE schedule. We reviewed the records of 50 patients treated as per our institutional protocols from 2007 to 2011. Two schedules of chemotherapy followed were vincristine, Adriamycin, cyclophosphamide (VAC) and VAC with alternate ifosfamide, etoposide (VAC + IE). Factors predictive of local failure and distant recurrence were analyzed. A total of 50 patients were analyzed. The median age at diagnosis was 14 years. Thirty-two patients were male, whereas 18 were female. Approximately, $95 \%$ of the patients relapsed after a median time gap of 1.6 years. The median 5 -year disease-free survival was $30 \%$. Systemic treatment with VAC or VAC + IE-based chemotherapy had equal local control and distant control rates. Smaller tumors had a better local control and lesser systemic failure than those

\section{Keywords}

- distant recurrence

- Ewing's sarcoma

- local recurrence

- pediatric cancer of larger sizes. Successful treatment of Ewing's sarcoma requires optimal systemic and local therapy. Both the chemotherapy regimens showed equal survival rates. Control of both the local and distant diseases is a result of the combined modality approach. Stage at presentation is the most important factor for prognosis. Complete surgery and local radiotherapy are important predictive factors for local and systemic control.
\end{abstract}

\section{Introduction}

The term Ewing's sarcoma family of tumors (ESFTs) defines a group of small round cell neoplasm of neuroectodermal origin that manifests as a continuum of neurogenic differentiation. ESFTs comprise $3 \%$ of all pediatric malignancies and represent the second most common malignant bone tumor in children and adolescents, with an estimated incidence in white children $<15$ years of age of 2.8 per 1 million. ${ }^{1}$ The last three decades have witnessed a major improvement in the outcome of ESFT patients.

DOI https://doi.org/ $10.1055 / \mathrm{s}-0039-1680842$ ISSN 2454-6798.
These advances in the treatment of ESFT have derived largely from cooperative trials and the progress made in the multidisciplinary approach. American and European studies have complemented each other in defining the active agents and their best schedules and combinations; new agents have been incorporated progressively into the treatment armamentarium, and improvements in support measures have allowed for treatment intensification. With most modern treatment regimens, the disease-free survival (DFS) for patients with localized disease may approach $70 \%$, whereas the overall survival (OS) may be $>80 \% .^{1-3}$ These

(C)2018 Spring Hope Cancer Foundation \& Young Oncologist Group of Asia
License terms

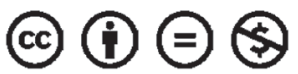


advances, however, are also the result of improvements in local control, with better radiation therapy (RT) planning and more aggressive surgical approaches. ${ }^{3}$ The optimization of local and systemic therapies for ESFT cannot be analyzed separately as both components are intrinsically intertwined; improvements in local and systemic control may impact each other. We have performed a comprehensive analysis of the St. Jude studies that represent the improvements made over the recent treatment eras and have evaluated the impact made by the different systemic and local control measures on outcome.

\section{Materials and Methods}

\section{Patients and Treatment}

A total of 50 patients were included in this retrospective study from 2007 to 2011. Patients with ESFT were treated with two schedules of chemotherapy: vincristine, adriamycin, cyclophosphamide (VAC) and VAC with alternate ifosfamide and etoposide (VAC + IE) or with radiotherapy along with surgery. $20 \%$ of patients had tumor in femur, $18 \%$ in ribs, and $16 \%$ in tibia ( - Table 1 ). The size of the tumor varied from 10 to $15 \mathrm{~cm}$ in $48 \%$ patients (-Table 2 ). The surgery involved $\mathrm{R} 0$ and $\mathrm{R} 12$ resections in 10 and 8 patients, respectively. The remaining patients underwent only biopsy. All patients had rib Ewing's sarcoma. Twenty-nine patients received six to eight cycles of VAC chemotherapy and 21 patients received VAC + IE. Radiotherapy involved a dose of 45 to $54 \mathrm{~Gy}$.

\section{Statistical Analysis}

OS was defined as the time interval from the date of diagnosis to the date of death from any cause or to last follow-up date. Event-free survival (EFS) was defined as the time interval from the date of diagnosis to the date of disease progression, recurrence, second malignancy, or death from any cause, whichever

Table 1 Patients characteristics: tumor site

\begin{tabular}{|l|l|l|}
\hline Site & Patients & $\%$ \\
\hline Head and neck & 4 & 8 \\
\hline Scapula & 5 & 10 \\
\hline Rib & 9 & 18 \\
\hline Humerus & 4 & 8 \\
\hline Pelvis & 8 & 16 \\
\hline Femur & 10 & 20 \\
\hline Tibia & 3 & 6 \\
\hline Spine & 3 & 6 \\
\hline
\end{tabular}

Table 2 Patient's characteristics: tumor size

\begin{tabular}{|l|l|l|}
\hline Size $(\mathrm{cm})$ & Patients & $\%$ \\
\hline$<5$ & 2 & 4 \\
\hline 5 to $<10$ & 19 & 38 \\
\hline 10 to $<15$ & 24 & 48 \\
\hline 15 to $<20$ & 3 & 6 \\
\hline 20 or $>20$ & 2 & 4 \\
\hline
\end{tabular}

occurred first, or to date of last follow-up for patients without events. OS and EFS distributions were estimated using Kaplan-Meier method. Local failure was defined as the time interval from the date of diagnosis to the date of local or regional recurrence or progression. Distant failure was defined as the time interval from date of diagnosis to date of distant disease recurrence or progression. Patients with both local and distant recurrences were included as having local recurrence for the analysis of local control and as having distant failure for the analysis of distant disease control.

\section{Results}

A total of 50 patients ( 32 males and 18 females) were analyzed. The median age at diagnosis was 14 years. Ninety-five patients relapsed at a median of 1.6 years. The 5 -year DFS rate was $30 \%$. On completion of treatment, 27 patients had complete response (CR), 17 had partial response (PR), and 6 had progression of disease (PD). On first follow-up, 4 patients had local recurrence. 15 had distant metastasis, with being the most common site. At last follow-up, 20 patients had CR, 6 had stable disease (SD), and 24 had PD. Intensifying the systemic treatment improved the local control. Smaller tumors had better local and systemic control than tumor of large sizes. Both chemotherapy regimens had nearly equal effect on survival $(p=0.510)$. Only 10 patients had $3 / 4$ grade of neutropenia. None of the patients had grade $3 / 4$ radiotherapy reactions. Complete surgical excision had better outcome than incomplete excision. Both male and female patients had equal survival rate. Patients with head and neck tumors had better survival than those with axial and peripheral tumors (-Figs. 1-4).

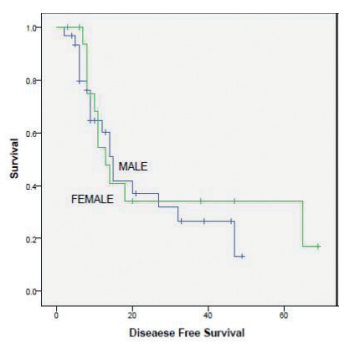

Fig. 1 Survival curve male versus female.

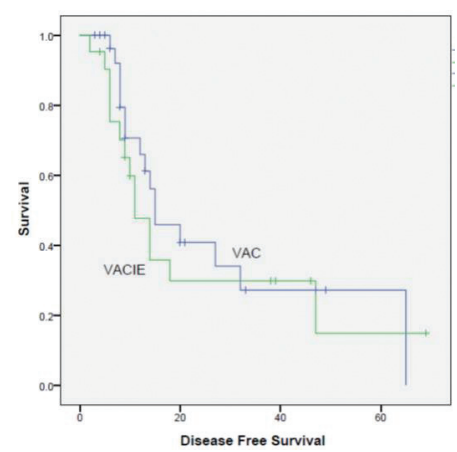

Fig. 2 Survival curve chemotherapy VAC (vincristine, Adriamycin, cyclophosphamide,) versus VAC + IE (VAC with alternate ifosfamide, etoposide). 


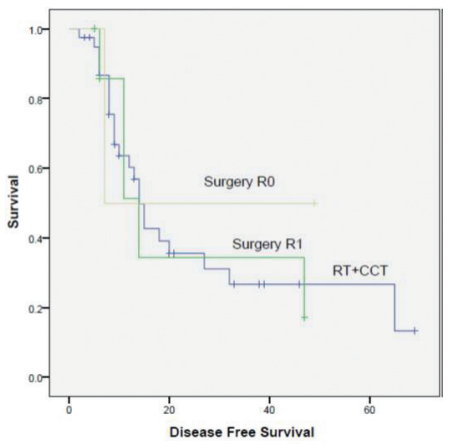

Fig. 3 Survival curve surgery, R0, R1, radiotherapy (RT), chemotherapy. CCT, concurrent chemotherapy.

\section{Discussion}

Patients who received definitive radiation for local control decreased progressively, and now most patients receive surgery or surgery plus radiation or radiotherapy with systemic chemotherapy. The impact made by improvements in local control appears to be greater than advances in systemic and local therapies. The importance of achieving good local control cannot be overemphasized; outcome after local failure is very poor. ${ }^{4}$ The 5 -year cumulative index (CI) of local failure was 25.1 to $63 \%$. Older patients, patients with large tumors, and patients with pelvic primaries had higher Cls of local failure. Local control, however, was also significantly influenced by treatment variables. First, and more importantly, the type of local control influenced outcome; combined treatment with surgery and RT resulted in lower $\mathrm{CI}$ of local failure than surgery alone, and surgery alone resulted in lower $\mathrm{Cl}$ of local failure than radiation alone. Available data indicate superior outcome with wide local surgical excision, although bias exists in selecting smaller, more peripheral tumors for definitive surgical resection. With careful selection for surgical therapy, local failure rates are $<10 \%{ }^{5-7}$ When surgery is used, the effect of tumor size on local failure is less clear. The combined Cooperative Ewing's Sarcoma Studies (CESS) and European Intergroup Cooperative Ewing's Sarcoma Studies did not demonstrate a difference in local failure when surgery was used for tumors $<100$ versus $100 \mathrm{~cm}^{3}$ (25.0 vs. $63.0 \%$, respectively). ${ }^{6}$ Postoperative and, more recently, preoperative irradiations have been applied to patients with marginally resected or poorly responding tumors. Despite the selection bias of unfavorable patients treated with combined local therapy, our data and the available literature indicate that there is an equivalent local control compared with surgery alone. ${ }^{6,8}$ The ES79 and ES87 studies added information on the use of low-dose adjuvant RT. ${ }^{9,10}$ The selection of low-dose irradiation has usually been on the basis of young age or favorable tumor characteristics such as small primary size and good response to chemotherapy. Although some centers have reported low local failure rates, low radiation doses are associated with suboptimal local control. ${ }^{6,9,11}$ Local failure rates for patients managed with RT

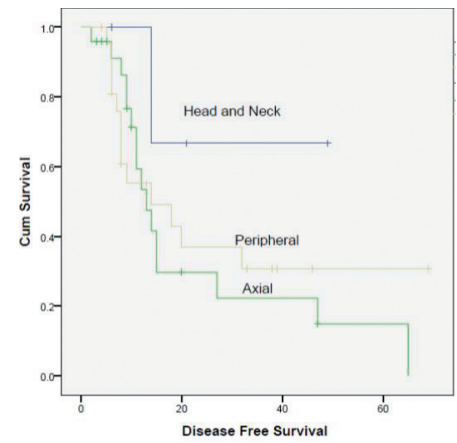

Fig. 4 Survival curve peripheral, axial, head and neck, and axial.

are consistently higher than those undergoing surgery, with recurrence rates of $30 \% .^{5-7}$ Local outcomes for our patients were positively influenced by age $<14$, tumor size $<10 \mathrm{~cm}$, and radiation dose $>45 \mathrm{~Gy}$. The improvements on the quality of radiation planning and delivery over the years, however, must be considered. Three cooperative group studies have demonstrated the importance of quality RT (CESS 81 and 86 and POG 8346). Central treatment plan review was instituted in CESS 86 following a local failure rate of 50\% in CESS 81 for patients undergoing definitive irradiation; subsequent patients treated with definitive RT on CESS 86 had a local failure rate of only $13 \% .^{9}$ Patients undergoing definitive radiation on POG 8346 had an $84 \%$ incidence of local failure if a major deviation in dose or volume of treatment was noted. Even patients with minor deviation experienced a $52 \%$ local failure rate compared with $20 \%$ for those with no deviation $(p=0.005) .{ }^{5}$ It is therefore very important to analyze the high historical incidence of local failure rates in patients treated with definitive radiation in the context of evolving and improving techniques. An important concept confirmed is that systemic therapy also influences local control. In patients who received treatment with definitive radiation with doses $>40 \mathrm{~Gy}$, the incorporation of chemotherapy resulted in better local control rates. It is certainly possible that the improved radiation planning and delivery of the later studies is a confounding factor. Other studies, however, support the notion that systemic therapy impacts local control. The DFS rate at 24 months is $30 \%$. Distant failure was associated with the presence of metastatic disease at diagnosis, which remains the most important prognostic factor. ${ }^{12}$ There is, however, some heterogeneity in patients with metastases. With an appropriately intensive treatment, patients with isolated lung metastases may have a better prognosis, albeit still worse than patients with localized disease, whereas patients with extrapulmonary metastases have a worse prognosis..$^{13}$ Distant failures were also associated with large primaries; tumor cell burden probably correlates with micro metastatic disease. With the use of molecular techniques in the staging of ESFT, it is evident that a proportion of patients with localized ESFT (20-40\%) have micro-metastatic disease measured as molecular detection of tumor cells by RT-PCR in peripheral blood or bone marrow. ${ }^{14}$ Finally, to evaluate 
the impact of local treatment measures on the control of the distant disease, we compared the incidence of metastatic recurrence among the different local treatment modalities. There was a trend toward better systemic control for patients treated with more aggressive local therapies. In summary, successful treatment of ESFT requires a very judicious use of intensive chemotherapy and aggressive local measures. Both treatment modalities are intertwined, and the control of the local and the distant disease is the result of the combined approach.

\section{Conflict of Interest}

None declared.

\section{References}

1 Marina NM, Pappo AS, Parham DM, et al; Jude Children's Research Hospital. Chemotherapy dose-intensification for pediatric patients with Ewing's family of tumors and desmoplastic small round-cell tumors: a feasibility study at St. Jude Children's Research Hospital. J Clin Oncol 1999;17(1):180-190

2 Craft AW, Paulussen M, Douglas C, et al. EICESS 92-early results of an international Ewing's tumour study. Med Pediatr Oncol 2000;35:191

3 Rodriguez-Galindo C, Spunt SL, Pappo AS. Treatment of Ewing sarcoma family of tumors: current status and outlook for the future. Med Pediatr Oncol 2003;40(5):276-287

4 Rodriguez-Galindo C, Billups CA, Kun LE, et al. Survival after recurrence of Ewing tumors: the St Jude Children's Research Hospital experience, 1979-1999. Cancer 2002;94(2):561-569

5 Donaldson SS, Torrey M, Link MP, et al; Pediatric Oncology Group. A multidisciplinary study investigating radiotherapy in Ewing's sarcoma: end results of POG \#8346. Int J Radiat Oncol Biol Phys 1998;42(1):125-135

6 Schuck A, Ahrens S, Paulussen M, et al. Local therapy in localized Ewing tumors: results of 1058 patients treated in the CESS 81, CESS 86, and EICESS 92 trials. Int J Radiat Oncol Biol Phys 2003;55(1):168-177
7 Bacci G, Forni C, Longhi A, et al. Long-term outcome for patients with non-metastatic Ewing's sarcoma treated with adjuvant and neoadjuvant chemotherapies. 402 patients treated at Rizzoli between 1972 and 1992. Eur J Cancer 2004;40(1):73-83

8 Shankar AG, Pinkerton CR, Atra A, et al; United Kingdom Children's Cancer Study Group (UKCCSG). Local therapy and other factors influencing site of relapse in patients with localised Ewing's sarcoma. Eur J Cancer 1999;35(12):1698-1704

9 Dunst J, Sauer R, Burgers JM, et al. Radiation therapy as local treatment in Ewing's sarcoma. Results of the Cooperative Ewing's Sarcoma Studies CESS 81 and CESS 86. Cancer 1991;67(11):2818-2825

10 Merchant TE, Kushner BH, Sheldon JM, LaQuaglia M, Healey JH. Effect of low-dose radiation therapy when combined with surgical resection for Ewing sarcoma. Med Pediatr Oncol 1999;33(2):65-70

11 Krasin MJ, Davidoff AM, Rodriguez-Galindo C, et al. Definitive surgery and multiagent systemic therapy for patients with localized Ewing sarcoma family of tumors: local outcome and prognostic factors. Cancer 2005;104(2):367-373

12 Cotterill SJ, Ahrens S, Paulussen M, et al. Prognostic factors in Ewing's tumor of bone: analysis of 975 patients from the European Intergroup Cooperative Ewing's Sarcoma Study Group. J Clin Oncol 2000;18(17):3108-3114

13 Paulussen M, Ahrens S, Burdach S, et al; European Intergroup Cooperative Ewing Sarcoma Studies. Primary metastatic (stage IV) Ewing tumor: survival analysis of 171 patients from the EICESS studies. Ann Oncol 1998;9(3):275-281

14 Schleiermacher G, Peter M, Oberlin O, et al; Société Française d'Oncologie Pédiatrique. Increased risk of systemic relapses associated with bone marrow micrometastasis and circulating tumor cells in localized Ewing tumor. J Clin Oncol 2003;21(1):85-91 